\title{
THE IMPORTANCE OF SSH RESEARCH IN HORIZON EUROPE
}

\author{
JOHN STEPHEN BELL \\ DOI: $10.22163 /$ fteval.2019.366
}

\section{INTRODUCTION}

$\mathrm{T}$ This paper is a revision of the proposals for the regulation and specific programme of the forthcoming European Framework Programme for Research and Innovation presented by the European Commission (EC) on 7 June 2018. It presents ideas on how Social Sciences and Humanities (SSH) research could be better integrated and puts forward suggestions for collaborative research and innovation as a main line of engendering change and securing competitiveness. It is crucial for the future of the "European Research Area" to recognise the value and importance of the SSH, including through continued annual SSH Monitoring Reports that have up to now illustrated the lack of progress that the EC has made in integrating SSH in Horizon 2020.

By engaging with the concepts of innovation and impact, the paper promotes an understanding of innovation as a factor to transform society and calls for a conceptualisation of impact that is taking wider social, cultural and political developments into account. Last but not least, this is followed by some practical suggestions for potential missions and ways of implementation.

\section{THE CONCEPTS OF "INNOVATION" AND "IMPACT"}

Horizon Europe brings together the European Union's (EU) research and innovation activities largely under one Framework Programme. But there is always a danger that the emphasis on the contribution of research to economic growth fosters a technocratic paradigm in which the translation of fundamental research into innovative 'products' is seen as the benchmark of success. In the past, the EC has understood the relationship between research and innovation too much in terms of an overly simplistic, linear process in which research is expected to lead to ever higher Technology Readiness Levels (TRLS). The dominance of this paradigm belittles the contribution of Humanities and the Social Sciences. Humanities and Social Sciences have different perspectives on problems, but they contribute to a rounded approach.

What is it that the Humanities contribute to innovation? They offer an ability to challenge present ways of approaching social problems by tes- ting out alternatives in rigorous, but non-experimental forms. Philosophy offers the chance to think through hypothetical alternatives, whilst literature makes use of imagination to conceive alternative scenarios and to explore them. Historians and archaeologists use the analysis of the past as a form of laboratory of different worlds. Being able to think through "what if?" scenarios deepen one's understanding of the world.

These approaches also bring into consideration the non-material features of our human existence. The quality of life depends not on having new gadgets or new products, but on being able to live a life which has value that may make use of what technology has to offer in a valuable way. Vision, beauty, style, and enjoyment are integral to a valuable human life.

The social sciences offer yet different ways of challenging contemporary norms and traditions of doing things. Techniques such as modelling enable alternative scenarios to be built and tested in a rigorous way without the ability to repeat experiments as in laboratory science. Modelling often reduces complexity by focusing on key features of a situation and then varying them.

Quantification is typically an approach of economics, geography and sociology. Other approaches look at qualitative analysis, scaling up from samples. These social sciences enable us not just to gain information about what might change, but also identify and test our deep values against which to test the social contribution of technological advances.

In our view, any worthwhile science programme for the EU has to harness the potential of all branches of scientific endeavour and to encourage them to work together. This perspective agrees with the view of the Lamy Report: "Innovation is more than technology. EU innovation policy must be based on a definition of innovation that acknowledges and values all forms of new knowledge - technological, but also business model, financing, governance, regulatory and social - which help generate value for the economy and society and drive systemic transformation."

"Innovation" should be redefined and implemented more holistically and openly in order to achieve the aims the EU wishes to support. Innovation is not limited to business and economic opportunities, but it is also fundamentally about transforming the way we live and the things we do, socially and culturally as well as economically. The humanities and social sciences have a very strong contribution to make such transformations happen.

Similarly, "impact" should be conceived in terms of how it affects not only the economy and governmental policies, but also the way social in- 
teractions, culture and ways of thinking are affected. There are good examples of this being recognised in Societal Challenge 6 of Horizon 2020, but the approach is not reflected consistently across the other Challenges. A definition of impact that incorporated the contribution of research and innovation to the wellbeing of society would be a significant and important step in the right direction.

The ambition of European research needs to look at how the individual and collective lives of residents in the EU are improved and how Europe can contribute to the quality of life of other parts of the world. Research needs to look at not only individual situations, but also the features of structures within society - power, institutions, political participation, and new actors in civil society. It explores cohesion and diversity in the way we are living together. It needs to investigate appropriate foundations and ingredients of contemporary democracy to make our societies more sustainable, open and resilient; widen our knowledge on the social and cultural dynamics and effects of (democratic) governance structures as we take advantage of changes that science and technology bring and the new questions they raise. For instance, fields of inquiry and more concrete objectives could include the social application of historical studies.

What is an appropriate balance between individuality and solidarity for modern European societies? Social and cultural diversity are valuable features of life in Europe (as in many other parts of the world). This provides not only a context for research and policy, but it also provides a wonderful resource. For example, if we look at the arts and society, we might ask whether literary models can enhance social cohesion?

Literature and art offer us a laboratory of the future, drawing on our deep sense of identities in the present and related to our past. Developing strategies to foster social access to art history and to critique, and to increase participation in cultural and artistic endeavour have creative potential to contribute to the transformation of society as much as any technological innovation.

\section{THE DESIGN OF THE EUROPEAN RESEARCH AGENDA}

In order to tackle the global challenges of the decades to come and enable European citizens and societies maintain the pace of innovation and social transformation, Europe needs to harness the creative capacities of all its researchers and social actors. This can be only achieved in a joint endeavour, especially by intensified inter- and transdisciplinary cooperation. In order to understand the human dimension of social and technological transformation, it is crucial to look at different perspectives and use the potential of the humanities and social sciences in enabling innovation and reflection. Each branch of science has its own contribution to knowledge and Horizon Europe needs to draw on them all in a holistic way.

This has implications for the drafting of the "Global Challenges" and "Missions". How do we identify the problems which these instruments are designed to solve and the methods appropriate for tackling them? The challenges Europe and its citizens face today and in the decades to come are not merely economic, technological and political, they are also social, cultural, legal and ethical. Challenges such as rising inequalities, nationalism, radicalism and terrorism threaten inclusion, social cohesion and democratic governance all over Europe. Demographic change, migration and digitisation create constant change. These challenges call for a profound and inclusive dialogue between all actors in society.

Technological innovation is obviously necessary to improve many features of the way we live, e.g. in medical interventions for healthcare, in smart systems to improve the quality of life, in ways of reducing threats to the climate and in improved transport. But technologies need to be embedded in an understanding of how we human beings might use them, how lives might adapt to their presence, and whether this would improve the quality of our lives. Scientists understand this well - they are, after all, members of society with a humane interest in living and contributing to good lives. They are keen to involve different branches of knowledge in ensuring that their efforts really do transform the lives of people in society.

From the perspective of the humanities and social sciences communities, these challenges require concerted efforts within and outside Europe, cutting across borders, cultures, languages, disciplines, sectors and institutions. That is why not only the "ALLEA Working Group Horizon Europe" argues for more interdisciplinarity and a bigger and well-defined role of the SSH in design and evaluation of the research which is funded through Horizon Europe. Otherwise the societal challenge to build inclusive, innovative and reflective societies runs the danger of being marginalised by other, more tangible material and technological challenges.

\section{MISSIONS}

The ambition of "Missions" to achieve tangible results within a defined timeframe is laudable in many ways. Nevertheless, again, it is necessary to guarantee interdisciplinarity and a bigger and well-defined role of the SSH in design and evaluation of the missions. In a statement published in cooperation with other stakeholders ${ }^{3}$, the ALLEA Working Group Horizon Europe critically reflects on the type and scope of missions that would adequately respond to the societal challenges Europe faces in the years and decades to come.

The 2030 Agenda of the United Nations (UN) should serve as a framework of inspiration when targeting these challenges through the development of missions. Such "Missions" have a strong potential to bring together researchers from many disciplines as well as political, cultural, economic and social actors and civil society in a common endeavour of ensuring that Europe is at the forefront of research, innovation and smart implementation - and hence well equipped to answer urgent societal questions. Actions should be inter- or multidisciplinary and involve organisations in the cultural, economic or social sectors: Co-creation of research questions will allow the translation of societal needs into research and innovation and facilitate the translation of research results into smart applications and societal uptake.

If it is to achieve worthwhile results, mission-oriented research should thus

(1) be transformative in that it generates new knowledge and understanding,

(2) acknowledge that innovation is more than technology, 
(3) be broad enough and not too constrained on specific end-products through a premature identification of indicators of success or failure,

(4) be open to researchers to come up with projects of all sizes to produce innovative ideas (bottom-up approach),

(5) integrate all countries and regions in order to counter the research and innovation divide in the "European Research Area" and maintain openness towards collaboration with non-EU countries.

Many of these ideas are reflected in the Mazzucato report ${ }^{4}$ on which the "Draft Regulation" now draws. The Mazzucato report sought to find a way in which research and economic growth could be steered. As a result, its second criterion is that missions should be "targeted, measurable" and time-limited (now Article 7(3)(c) of the "Draft Regulation on Horizon Europe", p. 14), and its third criterion is that they should be ambitious, but realistic. That requires careful thought in terms of the design of mission calls. This is a top-down activity by EU institutions. Of course, the final criterion for missions is that they should be open to multiple, bottom-up proposals (now Article 7(3)(f)). That leaves an important scope for the initiative of individual researchers and innovators or groups of them. But those bottom-up proposals will come within a framework. This feature draws out a major area for thought about Horizon Europe - not just the content and the budget, but the process by which it is implemented, particularly at the level of the EC.

\section{IMPLEMENTATION}

Call design: We consider that the drafting of "Work Programmes for Challenges and Missions" should draw on researchers in different disciplines and different methodologies as well as on experts from civil society and the culture and economics sector. The wording of calls should reflect the need to draw on the full range of research capacity within Europe. That has not always been the case in Horizon 2020 (especially outside "Societal Challenge 6" SC6) and this is reflected in the low level of participation by SSH disciplines within those other challenges.

The idea of involving humanities and social sciences in planning is to enable topics to be identified correctly in the first place. Take a current example: In the "Work Programme" of SC4 (Smart, Green and Integrated Transport) for 2018-2020, there is a call in relation to "Harnessing and understanding the impacts of changes in urban mobility on policy making by city-led innovation for sustainable urban mobility" (LC-MG-1-3-2018). The detail of the challenge states that "Urban mobility is in transition. This is a result of, for example, changing user needs; emerging transport technologies; new transport services using new business models; and new institutional and financing structures." (LC-MG-1-3-2018, p.20). Further it states that "Special attention should be paid to the needs of vulnerable groups and users with different cultural backgrounds taking into account gender issues; and to the specific context of areas that are undergoing rapid economic change." (LC-MG-1-3-2018, p.20). Both of these clearly call for a contribution by social scientists and humanities scholars in order to understand the social needs that transport technologies, business models and financing structures are required to serve.
However, compared with the emphasis on data-driven planning, new business models and technology, not much thought has gone into identifying the social phenomena which research in this area should be addressing. It should also be looking at why people are using vehicles, how changes in work patterns (e.g. mobile and home working) affect demands for and timing of vehicle use, and whether the location of schools, leisure and shopping venues make a difference. In a document which runs to over a hundred pages on all the calls, the thought given to the potential contribution of humanities is very limited, and the contribution of social scientists, such as social geographers, is badly under-developed. There are words which have potential, but in comparison with the detail on other matters, they give the impression of being an after-thought.

Horizon Europe should aim to designate broad fields of enquiry which leave substantial flexibility to accommodate the innovative, but unexpected proposal. This means that the drafts of calls should be far less detailed than the current calls for "Societal Challenges" within Horizon 2020.

Emerging priorities: Given the uncertainty about the future and the rapid development of technology, the fields of research identified for "Missions" should not cover the whole of the 2021-2027 period, but should initially be shorter, with the possibility of continuation where they prove fruitful.

Project design: Projects submitted should be broad enough to include, where appropriate, participatory actions (co-design) by non-researchers. For example, research on migrants or elderly people might involve those groups in shaping the design of projects and in selecting the materials to be included as part of the research. It is in these ways that "citizen-led science" is best understood. Such processes of co-creation of knowledge ensure better acceptance and implementation in society and the economy. In many research projects in the humanities, it is common to bring together individuals from communities that are being studied to help design and implement the research through writing, oral history or articulating features of their communities which shape the understandings that are necessary for effective research.

Evaluation: The evaluation of proposals should include representatives of a range of disciplines, including the humanities and social sciences. The diversity of social science and humanities subjects (like the diversity of biological sciences) requires a range of expert evaluators to be involved to reflect the diversity of disciplines (and the emerging new fields generated through inter- and transdisciplinary collaboration). "Ethics Reviews" need informed experts.

Project size: There should be greater flexibility in choosing the size of a project. Projects in the humanities and social sciences typically do not have the need for expensive equipment. There are sometimes good reasons for larger teams, but often close working by smaller teams is the most productive way forward. The permitted size of bids should be smaller than in Horizon 2020. It may be prudent to give smaller amounts of initial funding until the proof of concept stage is reached or potential social or conceptual impact is envisaged. This calls for follow-up funding for promising ideas.

Monitoring: Review criteria should recognise that research/science accept a diversity of good solutions and a complexity of contexts in which solutions achieve results. New indicators for societal and cultural impact need to be developed and used. Assessing the performance of missions 
cannot simply be in terms of success (man on the moon) or failure (no man on the moon). Scholars from the humanities and social sciences need to be integral to the monitoring if the potential for steering research and social development (not just economic growth) is to be realised.

\section{CONCLUSION}

The humanities and social sciences need to be deeply embedded in the research agenda of Horizon Europe. This is because the research endeavour needs them in order to achieve its transformational potential. Researchers in these fields do have different research methods and ambitions compared with engineers and scientists, but this offers the potential for mutual enrichment. Ultimately, we are serving a community of nations within the EU who have the ambition to work together to improve their own quality of individual and community lives as well as those of the wider world. If we fail to do this effectively through Horizon Europe, we let down ourselves as researchers and the people we serve.

\section{REFERENCES}

ALLEA (2017). Developing a Vision for Framework Programme 9. Retrieved October 18, 2018 from: https://www.allea.org/wp-content/uploads/2017/07/ALLEA_Statement_FP9.pdf.

ALLEA et al. (2017). Living Together: Missions for Shaping the Future. Retrieved October 18, 2018 from: https://www.allea.org/wp-content/ uploads/2017/12/Living_Together_Missions_for_Shaping_the_Future_2017.pdf

High Level Group on maximizing the impact of EU Research \& Innovation Programmes (2017). LAB - FAB - APP - Investing in the European future we want, Report of the independent High Level Group on maximising the impact of EU Research \& Innovation Programmes. Retrieved October 18, 2018 from: http://ec.europa.eu/research/evaluations/pdf/ archive/other_reports_studies_and_documents/hlg_2017_report.pdf

LC-MG-1-3-2018, (2018). Harnessing and understanding the impacts of changes in urban mobility on policy making by city-led innovation for sustainable urban mobility: http://ec.europa.eu/research/participants/ data/ref/h2020/wp/2018-2020/main/h2020-wp1820-transport_en.pdf

Mazzucato, M. (2018). Mission-Oriented Research and Innovation in the European Union. Retrieved October 18, 2018 from: https://ec.europa.eu/ info/sites/info/files/mazzucato_report_2018.pdf.

\section{JOHN STEPHEN BELL}

Chair of the ALLEA Working Group Horizon Europe, All European Academies (ALLEA)

Jägerstraße 22/23, Berlin, 10117 (Germany)

E: jsb48@cam.ac.uk

\section{AUTHOR}

